

\title{
Full field imaging of isolated metallic nano objects
}

\author{
E. Absil, G. Tessier, D. Fournier, M. Gross, Michael Atlan
}

\section{To cite this version:}

E. Absil, G. Tessier, D. Fournier, M. Gross, Michael Atlan. Full field imaging of isolated metallic nano objects. European Physical Journal: Applied Physics, 2009, 47 (1), pp.1-4. 10.1051/epjap/2009023 . hal-00487525

\section{HAL Id: hal-00487525 \\ https://hal.science/hal-00487525}

Submitted on 30 May 2010

HAL is a multi-disciplinary open access archive for the deposit and dissemination of scientific research documents, whether they are published or not. The documents may come from teaching and research institutions in France or abroad, or from public or private research centers.
L'archive ouverte pluridisciplinaire HAL, est destinée au dépôt et à la diffusion de documents scientifiques de niveau recherche, publiés ou non, émanant des établissements d'enseignement et de recherche français ou étrangers, des laboratoires publics ou privés. 


\title{
Full field imaging of isolated metallic nano objects
}

\author{
E. Absil, G. Tessier, and D. Fournier \\ Ecole Supérieure de Physique et Chimie Industrielles de la Ville de Paris \\ CNRS UPR5, Université Pierre et Marie Curie - Paris 6, \\ 10 rue Vauquelin 75231 Paris cedex 05. France \\ gilles.tessier@espci.fr \\ M. Gross and M. Atlan \\ Ecole Normale Supérieure, CNRS UMR 8552, \\ Université Pierre et Marie Curie - Paris 6, \\ 24 rue Lhomond 75231 Paris cedex 05. France
}

(Dated: November 3, 2008)

\begin{abstract}
Since the detection of nanoparticles is a major issue in nano sciences, we have developed two instruments for the fast imaging of individual nano objects. The first one is based on a spatial modulation of the sample using an incoherent dark field illumination and a multiplexed lock in detection. The second instrument uses digital heterodyne holography. Both instruments make it possible to achieve a high signal-to-noise ratio and therefore to detect very low signal.
\end{abstract}

PACS numbers: 78.67.Bf 78.90.+t 


\section{INTRODUCTION}

Detecting nano objects has been a subject of great interest for the last ten years because applications are numerous in nano sciences [1] [2] [3] and biology [4] [5]. As the optical properties of the nano objects strongly depend on their size, shape and environment, far field optical measurements allow a good characterization. Furthermore, detecting isolated nano objects prevents from any averaging the optical information and gives access to local optical properties.

Today, most biological labels are luminescent. At the beginning, fluorescent dyes were heavily used but were limited by their photobleaching that induces a short life time. To increase the life time, semi conductor nano crystals have been developed but also appear to bleach and blink, which yields an instability of the detected signal with time. To circumvent this issue, we propose to detect metallic nano objects in our experiments, for further implementation as biological labels.

In the last five years, different far field techniques based either on the absorption [6] [7] or on the scattering of light by the particles have been developed. Most of these methods use a single detector that implies a long acquisition time because of the scanning of the sample and prevents from observing moving objects. In order to dramatically reduce the acquisition time, we propose different instruments providing wide field measurements owing to a multi pixel detector.

The power of the detected signal is proportional to the power of the source and to the cross section of the particle. As the scattering cross section of a spherical particle scales up quadratically with the volume of the particle, the signal is very low for small particles and this constitutes the main difficulty in our experiments. To be able to detect nano objects, the continuous background has to be reduced and the sensitivity maximized. Thus, we propose two different instruments using either a dark field or an off-axis illumination to avoid stray light, and combined with a multiplexed lock in detection to extract the relevant signal modulated either by a spatial modulation of the sample or by a phase shift between the interfering beams. 


\section{FULL FIELD IMAGING BASED ON A SPATIAL MODULATION OF THE SAMPLE}

This technique uses a dark field illumination [8] in order to reduce stray light. Thus, only the light scattered by the sample penetrates in the accepting angle of the microscope objective. With no further implementation, particles with diameter lower than $40 \mathrm{~nm}$ can not be detected [9]. That is why, to modulate the signal, the position of the sample is modulated in the plane perpendicular to the optical axis. In order to extract only the modulated part of the detected signal, this system is combined with a multiplexed lock in detection [10]. By using the proper signal processing, the non-modulated part of the image (defects of the camera and of the illumination) is cancelled.

As the particles are illuminated with an incoherent source, this technique also allows acquisition of the full scattered-light spectrum of an individual particle.

\section{A. Experimental set-up}

The sample is obtained by electronic lithography. It consists in gold dots (height: $7 \mathrm{~nm}$, width: $60 \mathrm{~nm}$, spaced out $7 \mu \mathrm{m}$ ) placed in a matrix configuration on a silicon substrate. Fig.1 shows a diagram of the sample and of the experimental setup. The sample is lit by an incoherent source (100 W halogen lamp) in a dark field illumination configuration. The position of the sample is sinusoidally modulated at the frequency $f_{P Z T}$ in the plane perpendicular to the optical axis using a piezo electric actuator driven by the low frequency generator \# 2. To prevent overlapping of the signal when images are demodulated, the amplitude of the modulation is larger than the diffraction pattern of the dot, typically a few micrometers. The charge coupled device (CCD) camera, driven by generator \#1, is used in a four-buckets integration mode [10], i.e. it acquires four images per period of the spatial modulation: $f_{C C D}=4 f_{P Z T}=24 \mathrm{~Hz}$. Generators \# 1 and \# 2 share a common reference clock to avoid phase drifts between the spatial modulation and the camera acquisitions.

\section{B. Full field imaging of gold nano dots}

We acquired 100 images $i_{m}$ during $N=25$ periods of the spatial modulation $\left(T_{P Z T}=\right.$ $167 \mathrm{~ms}$ ) with an exposure time of $10 \mathrm{~ms} \ll \mathrm{T}_{\mathrm{PZT}}$. The signal processing on these images 


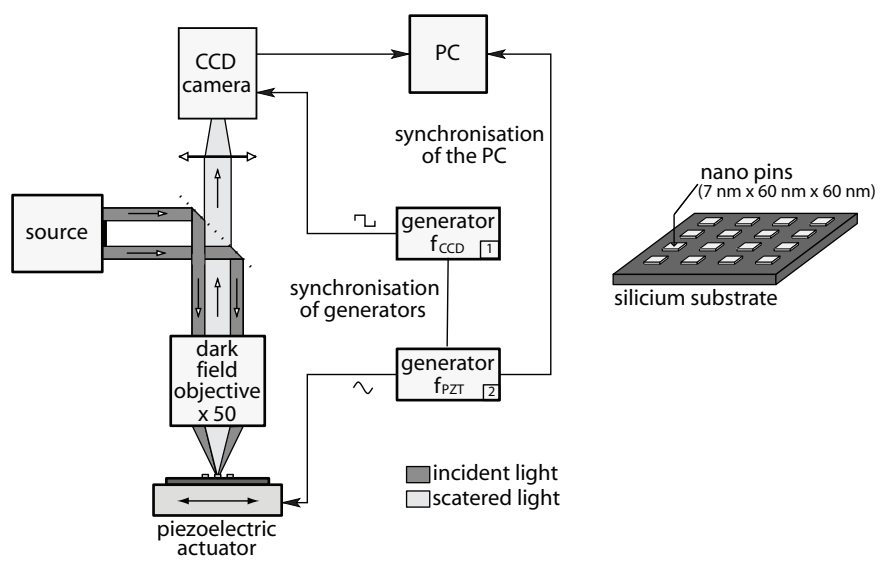

FIG. 1: Experimental set-up and zoom on the sample.

yields the amplitude image $I_{a m p}[10]$ :

$$
I_{a m p}=\left|\left(I_{1}-I_{3}\right)+j\left(I_{2}-I_{4}\right)\right|=\frac{\sqrt{\left(I_{1}-I_{3}\right)^{2}+\left(I_{2}-I_{4}\right)^{2}}}{4 N}
$$

where $j^{2}=-1$ and $I_{p}=\sum_{k=0}^{N-1} i_{4 k+p}$ with $p=1$ to 4 . Fig.2 shows the diagram of the matrix (a) sample (10x10 dots) observed and the acquired image (b) which are very similar except for a few dusts particles in the field of view. Due to the signal processing two spots correspond to one gold pin, as seen on the surface image of six dots Fig.2(c). If needed, this measurement artefact can be removed by a deconvolution of the image which is represented on Fig2(d).

Thus, with the described setup and signal processing, dots of $7 \mathrm{~nm}$ in thickness and $60 \mathrm{~nm}$ in width are easily detected. Neglecting the geometry effects, a dot can be roughly compared to sphere of the same volume, i.e. $36 \mathrm{~nm}$ in diameter, as the scattering cross section varies quadratically with the volume. Simulations of the interaction of light with a $36 \mathrm{~nm}$ spherical particle (Mie calculation of the scattering cross section using the refractive index of bulk gold [11] for the relevant range of wavelengths) and of the whole measurement process (spectral power of the source and quantum efficiency of the camera) show that this instrument based on a spatial modulation is able to detect a signal down to $0.04 \mathrm{pW}$, i.e. $10^{5}$ photons per second per frame, which is a very good sensitivity. 
(a)

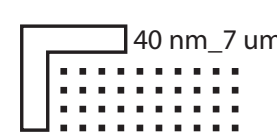

:a:a:
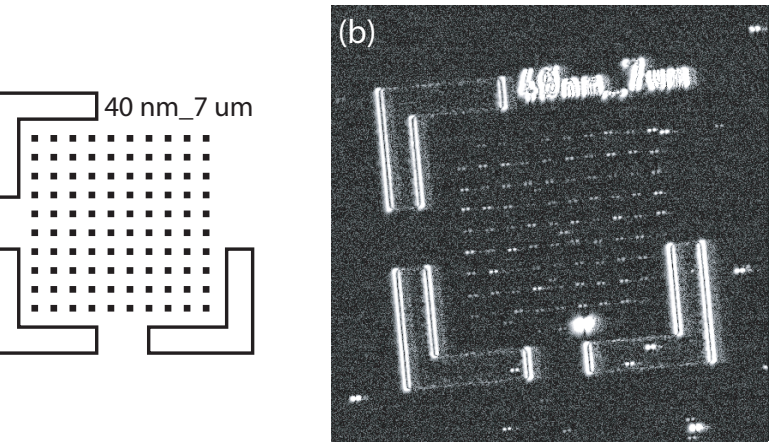

(c)

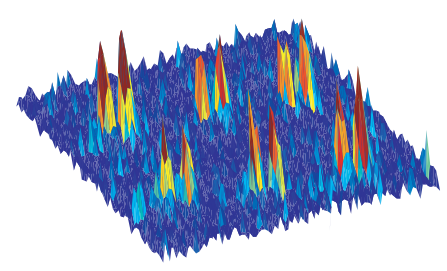

(d)

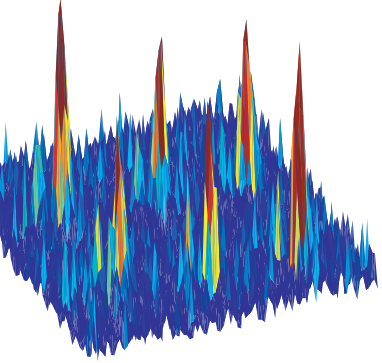

FIG. 2: Diagram of the sample (a) and acquired image in logarithmic arbitrary units (b). surface image of six dots before (c) and after (d) deconvolution.

\section{Towards spectroscopic characterization}

Owing to the technique based on a spatial modulation of the sample, which is an incoherent equivalent of the spatial modulation scanning imaging [7] combined with a multiplexed lock in detection [10], full scattering spectra of isolated nano objects should be obtained very soon by adding an imaging spectrometer between the microscope and the CCD camera.

\section{FULL FIELD IMAGING USING INTERFEROMETRY}

The second instrument we developed for the detection of isolated gold nano objects is based on digital heterodyne holography. The light from the incident laser beam which is scattered by the nano objects is collected by a microscope objective and propagates to the CCD camera. In the CCD plane, the wave coming from the sample interferes with a reference beam or local oscillator (LO) coming from the same laser source as the probing beam.

The novelty of this experiment does not lie in the instrument itself that have been well describes in references [13] and [12] but in the observation in three dimensions of the scattering diagram of a metallic nano-object. 


\section{A. Interferometric experimental setup}

The sample consists in $200 \mathrm{~nm}$ diameter gold nanoparticles (NPs) embedded in polyvinyl alcohol and spin coated on a glass slide in order to obtain less than one particle per $\mu \mathrm{m}^{2}$ to avoid any inconvenience caused by the diffraction-limited optical resolution. Fig.3 shows the experimental setup. The main laser beam at wavelength $\lambda=532 \mathrm{~nm}$, i.e in the plasmon resonance range of gold NPs, is split (BS) in two secondary beams.

The first beam $\left(E_{I}, f_{I}\right)$ illuminates the sample in a configuration very similar to dark field, as only the light scattered by the NPs, called the signal beam $\left(E_{S}, f_{S}\right)$, is collected by the microscope objective and reaches the CCD camera. At the same time, the secondary beam $\left(E_{L O}, f_{L O}\right)$, called reference or local oscillator, is expanded $(\mathrm{BE})$ and propagates to the camera with a tilt angle of approximately $1^{\circ}$ with respect to the average direction of propagation of the signal beam. In the plane of the CCD camera, the LO beam $\left(E_{L O}, f_{L O}\right)$ and the signal beam $\left(E_{S}, f_{S}\right)$ interfere and the corresponding interferometric pattern is called the hologram. The off-axis holographic configuration will allow a fast and simple filtering of the unwanted signal coming mainly from the LO [12].

The signal and the LO beams can be attenuated using neutral densities ND1 and ND2 in order to adjust their respective intensities and to optimize the total magnitude of the signal according to the dynamic range of the CCD camera.

In order to minimize the parasitic contributions of the LO and the twin image in the hologram, the interferometric pattern is modulated by shifting the optical frequency of the signal and LO beams using acousto optic modulators AOM1 and AOM2 [12] yielding $f_{S}=f_{L}+f_{A O M 1}$ and $f_{L O}=f_{L}+f_{A O M 2}$ respectively, where $f_{L}$ is the optical frequency of the laser. The phase shift between the two waves that interfere induces a beating of the hologram at the frequency $f_{H}=f_{A O M 2}-f_{A O M 1}$ that is set in order to acquire four images per period of the beating: $f_{H}=f_{C C D} / 4$, to extract the relevant part of the hologram. In order to avoid phase drifts the generators that drives the AOMs share a common reference clock. 


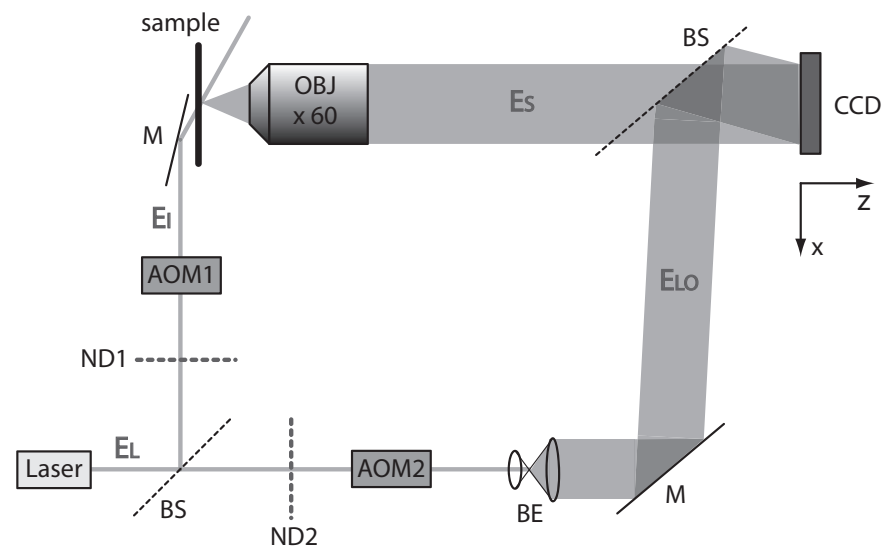

FIG. 3: Digital heterodyne holographic setup. BS: beam splitter, ND: neutral density, AOM: acousto-optic modulator, BE: beam expander, M: miror, CCD: charge coupled device camera.

\section{B. Full field imaging of gold NPs}

The virtual image is reconstructed owing to a double Fourier transformation (FT) process and to a numerical backward propagation of light through the demodulated hologram, in the plane $z=0$ of the CCD camera in the real space, obtained by summing the four images $I_{p}$ (calculated as in II B) with an appropriate phase factor:

$$
H(x, y, 0)=\sum_{m=0}^{3} j^{m} I_{m}=\left(I_{0}-I_{2}\right)+j\left(I_{1}-I_{3}\right)
$$

The hologram in the Fourier space is obtained by a 2D FT:

$$
\tilde{H}\left(k_{x}, k_{y}, 0\right)=F T[H(x, y, 0)]
$$

As the directions of propagation of the LO and signal beams are slightly tilted, the three diffraction orders (corresponding to the LO intensity ( 0 order), the twin image $(-1)$ and the virtual image $(+1)$ ) do not overlap in the Fourier space which allows a spatial filtering of the relevant signal $(+1)$ yielding the filtered Fourier space hologram $\tilde{H}_{f i l t}\left(k_{x}, k_{y}, 0\right)$.

The backward propagation of the scattered field at a distance $z$ in Fourier space is written:

$$
\tilde{H}_{f i l t}\left(k_{x}, k_{y}, z\right)=\tilde{H}_{f i l t}\left(k_{x}, k_{y}, 0\right) \cdot \exp \left(j \frac{k_{x}^{2}+k_{y}^{2}}{k} z\right)
$$

where $k=2 \pi / \lambda$ is the optical wave factor and the exponential factor is the kernel function that describes the Fourier space propagation from 0 to $z$. The reconstructed image in plane 
$z$ is then obtained by an inverse FT:

$$
H(x, y, z)=F T^{-1}\left[\tilde{H}_{f i l t}\left(k_{x}, k_{y}, z\right)\right]
$$

The image can be reconstructed in several $z$ planes in order to obtain a 3D image and most noticeably in the focal plane of the objective $z=z_{0}$.
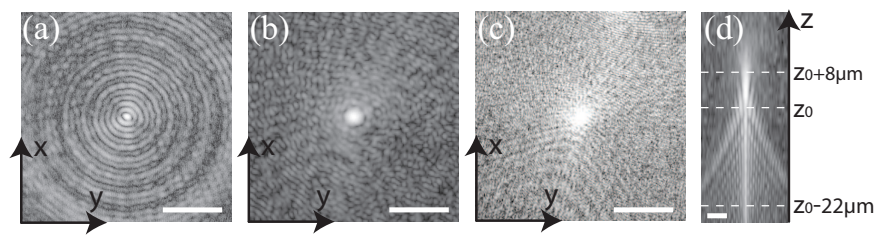

FIG. 4: Reconstructed images of a $200 \mathrm{~nm}$ gold NP at several axial positions: (a) $z=z_{0}-22 \mu \mathrm{m}$, (b) $z=z_{0}$ (focal plane of the objective), (c) $z=z_{0}+8 \mu \mathrm{m}$, displayed in logarithmic arbitrary units.(g) Axial plane distribution. Scale bar is $5 \mu \mathrm{m}$.

Fig.4 shows the reconstructed image of an isolated $200 \mathrm{~nm}$ gold nanoparticle in different transversal $z$ plane (a-c) and the axial plane distribution over $47 \mu \mathrm{m}(\mathrm{d})$. This result denotes a signal elongated by several microns in the axial direction. A particle of $200 \mathrm{~nm}$ in diameter has been used in this experiment to obtain a very good signal to noise ratio but smaller particles (down to $30 \mathrm{~nm}$ ) can be detected with this setup owing to its very high sensitivity of one photon per second per pixel [13].

\section{CONCLUSION AND PROSPECT}

The instrument we developed, based on a spatial modulation of the sample, allowed to detect nano dots with a volume equivalent to a $36 \mathrm{~nm}$ diameter sphere and showed a very good sensitivity of $10^{5}$ photons per second per frame. Spectroscopic abilities are expected to be implemented to this setup in order to obtain full scattered-light spectra of individual nano objects.

Digital holography in phase-shifting and off-axis configuration is a very sensitive technique ( 1 photon per second per pixel) and allows acquisition of the full diffusion diagram of a nano object (down to $30 \mathrm{~nm}$ ) by recording only one hologram. To detect smaller objects, photothermal effects will be implemented in this setup. 
The two instruments presented here show a similar sensitivity $\left(10^{5}\right.$ photons per second per frame for spatial modulation and $1,4.10^{5}$ for holography) and the holographic setup, unlike the spatial modulation setup, allows a fast 3D-imaging of nano-objects.

The authors acknowledge support from the Agence Nationale pour la Recherche (ANR 3D NanoBioCell) and the Centre de comptences NanoSciences Ile de France (C'nano IdF).

S. Janel and R. Boukherroub from the Institut d'Electronique de Microelectronique et de Nanotechnologie (IEMN, Lille, France) are also gratefully acknowledged for the conception of the sample obtained by electronic lithography.

[1] S. A. Maier, Current Nanoscience, 1, 17 (2005)

[2] F. Stellacci, C. A. Bauer, T. Meyer-Friedrichsen, W. Wenseleers, V. Alain, S. M. Knebler, S. J. K. Pond, Y. Zhang, S. R. Marder, J. W. Perry, Adv. Mater., 14, 194 (2002)

[3] T. Kalkbrenner, M. Ramstein, J. Mlynek, V. Sandoghdar, J. Microsc., 202, 72 (2001)

[4] D. Lasne, G. A. Blab, S. Berciaud, M. Heine, L. Groc, D. Choquet, L. Cognet, B. Lounis, Biophys. J., 91, 4598 (2006)

[5] S. Enoch, R. Quidant, G. Badenes, Optics Express, 12, 3422 (2004)

[6] S. Berciaud, D. Lasne, G. A. Blab, L. Cognet, B. Lounis, Phys. Rev. B, 73, 045424 (2006)

[7] A. Arbouet, D. Christofilos, N. Del Fatti, F. Valle, J. R. Huntzinger, L. Arnaud, P. Billaud, M. Broyer, Phys. Rev. Lett., 93, 127401 (2004)

[8] Murphy, B. Douglas, Fundamentals of Light Microscopy and Electronic Imaging, John Wiley and sons, New York (2001)

[9] D. Boyer, P. Tamarat, A. Maali, B. Lounis, M. Orritz, Science, 297, 1160 (2002)

[10] P. Gleyzes, F. Guernet, A. C. Boccara, J. Optics, 26, 251 (1995)

[11] P. B. Johnson, R. W. Christy, Phys. Rev. B, 6, 4370 (1972)

[12] M. Gross, M. Atlan, E. Absil, Applied Optics, 47, 1757 (2008)

[13] M. Gross, M. Atlan, Optics Letters, 32, 909 (2007) 